Review

\title{
STAMPing into Mitochondria
}

\author{
Seong Keun Yoo ${ }^{1,2}$, JaeHun Cheong1 and Hye Young Kim ${ }^{1 凶}$ \\ 1. Department of Molecular Biology College of Natural Sciences Pusan National University Busan 609-735, Korea; \\ 2. Department of Biological Sciences College of Life Science and Bioengineering Korea Advanced Institute of Science and Technology \\ Deajeon 305-701, Korea.
}

\begin{abstract}
$\square$ Corresponding author: Hye Young Kim. Phone, 82-51-240-2791; Fax, 82-51-241-3767; E-mail, dolph02@donga.ac.kr.
(c) Ivyspring International Publisher. This is an open-access article distributed under the terms of the Creative Commons License (http://creativecommons.org/ licenses/by-nc-nd/3.0/). Reproduction is permitted for personal, noncommercial use, provided that the article is in whole, unmodified, and properly cited.
\end{abstract}

Received: 2013.12.30; Accepted: 2014.02.25; Published: 2014.03.08

\begin{abstract}
Six transmembrane protein of prostate 2 (STAMP2) is a protein that has been extensively studied due to its association with prostate cancer. Currently, STAMP2 is well known for its critical role in metabolism and modulating inflammatory signals. Even so, the molecular mechanism of STAMP2 activity and its downstream effectors are still largely unknown. Here, we review the current knowledge of STAMP2, and suggest possible explanations for some of its less well-understood features. A few studies suggest that STAMP2 may interact with mitochondria. Considering STAMP2 functions as a potential component of mitochondrial biology may yield valuable insight into this protein.
\end{abstract}

Key words: STAMP2, mitochondria, metalloreductase, metabolism, inflammation, insulin resistance.

\section{Nomenclature}

The STAMP protein family has been studied using multiple different approaches. Due to its many unique features, the protein family is designated by several nomenclature systems. The protein family was initially named STEAP (six transmembrane epithelial antigen of the prostate) based on its potential use as prostate cancer marker (1). Other studies of prostate-specific antigens generated the designation STAMP (six transmembrane protein of prostate) (2). Hence, both the STAMP and STEAP designations are used regularly: STAMP1, STAMP2 and STAMP3 correspond to STEAP2, STEAP4 and STEAP3, respectively.

Independent studies of adipose conversion related to tumor necrosis factor $\alpha(\mathrm{TNF}-\alpha)$ in rats (Rattus norvegicus) confirmed the involvement of a six transmembrane cell surface protein, which was named TIARP (tumor necrosis factor a-induced adipose-related protein) (3). High homology between TIARP and its STEAP counterparts in humans (Homo sapiens) later led to it being renamed STEAP 4.

There are a few differences between TIARP,
STEAP4, and STAMP2. For example, unlike TIARP, STEAP4 does not appear to regulate preadipocyte differentiation in vitro (4), and it is important to study these species-specific differences. The STAMP2, STEAP4, and TIARP naming conventions are used interchangeably.

\section{Discovery of the STAMP2 protein}

Prostate cancer is one of the most common types of cancer among men in the western world. Androgens and the androgen receptor are extremely important in prostate carcinogenesis. However, the specific molecular mechanisms of prostate carcinogenesis are not fully understood, and identifying a prostate-specific antigen has proved to be a significant challenge (5).

In their search for androgen receptor-regulated genes, Korkmaz et al. (2) discovered a highly prostate-specific gene that they named STAMP1. BLAST analysis later identified at least 2 proteins with similar sequences, which were subsequently cloned, studied, and named STAMP2 and STAMP3 (6). 


\section{STAMP2 expression}

STAMP2 protein expression appears to be restricted to several tissues. Human STAMP2 is expressed in the lung, placenta, heart and prostate. It can also be detected at lower levels in the liver, testes, skeletal muscle, small intestine, and pancreas. Several studies suggest that STAMP2 is highly expressed in the adipose tissue and joints of patients with rheumatoid arthritis (RA) (6-8).

As mentioned earlier, TIARP, the mouse homo$\log$ of STAMP2, was first identified due to its dramatically increased expression in adipocytes in response to TNF-a (3). Stamp2 expression is also highly up-regulated in the murine cell line 3T3-L1 during differentiation of preadipocytes into adipocytes (9). STAMP2 mRNA is expressed at high levels in both white adipose tissue (WAT) and brown adipose tissue (BAT). STAMP2 mRNA levels in human WAT are strongly associated with enlarged fat cells and insulin resistance but do not play a protective role against insulin resistance (10).

\section{STAMP2 structure and function}

STAMP2 is located on chromosome 7q21 and contains 5 exons and 4 introns. The genomic sequence is relatively small (approximately $26 \mathrm{~kb}$ ) because intron 1 is extremely large $(22,516 \mathrm{bp})$. The gene is transcribed into a single $4.0 \mathrm{~kb}$ mRNA with a 5 '-untranslated region (UTR) of approximately $1.7 \mathrm{~kb}$ (6). The protein is comprised of 495 amino acids and contains 6 transmembrane regions near the
C-terminal domain. The N-terminal domain contains 3 conserved motifs: a dinucleotide binding domain, an NADP oxidoreductase motif and a motif similar to pyrroline 5-carboxylate reductase (Figure 1) (6).

The metalloreductase activity of STAMP family proteins was first identified in STAMP3 (STEAP3). Positional cloning of the iron deficient mouse mutant nm1054 identified STAMP3 (STEAP3) as the responsible gene. BLAST analysis shows that STAMP3 shares homology with $\mathrm{F}_{420} \mathrm{H}_{2}$ : NADP+ oxidoreductase (FNO) and the FRE family of yeast metalloreductases. Overexpression of STAMP3 also stimulates erythroid ferrireductase activity. STAMP3 acts not only as reductase but also stimulates iron and copper uptake. Taken together, these features suggest that STAMP3 is an endosomal ferrireductase (11).

A later study showed that STEAP, STAMP1, and STAMP2 have similar functions. In situ hybridization of mouse embryo sections shows a tissue expression pattern relevant to metal homeostasis. In addition, these proteins co-localize with transferrin and transferrin receptor, which are vital components of the transferrin (Tf) cycle. Furthermore, transient expression of the STAMP proteins in HEK-293T cells revealed that they have ferririductase and cupric reductase activity (12).

The crystal structure of the murine Stamp2 oxidoreductase domain was solved recently. The structure suggests that STAMP2 utilizes an interdomain flavin-binding site to shuttle electrons between the NADPH-utilizing oxidoreductase domain and the transmemebrane heme group (13).

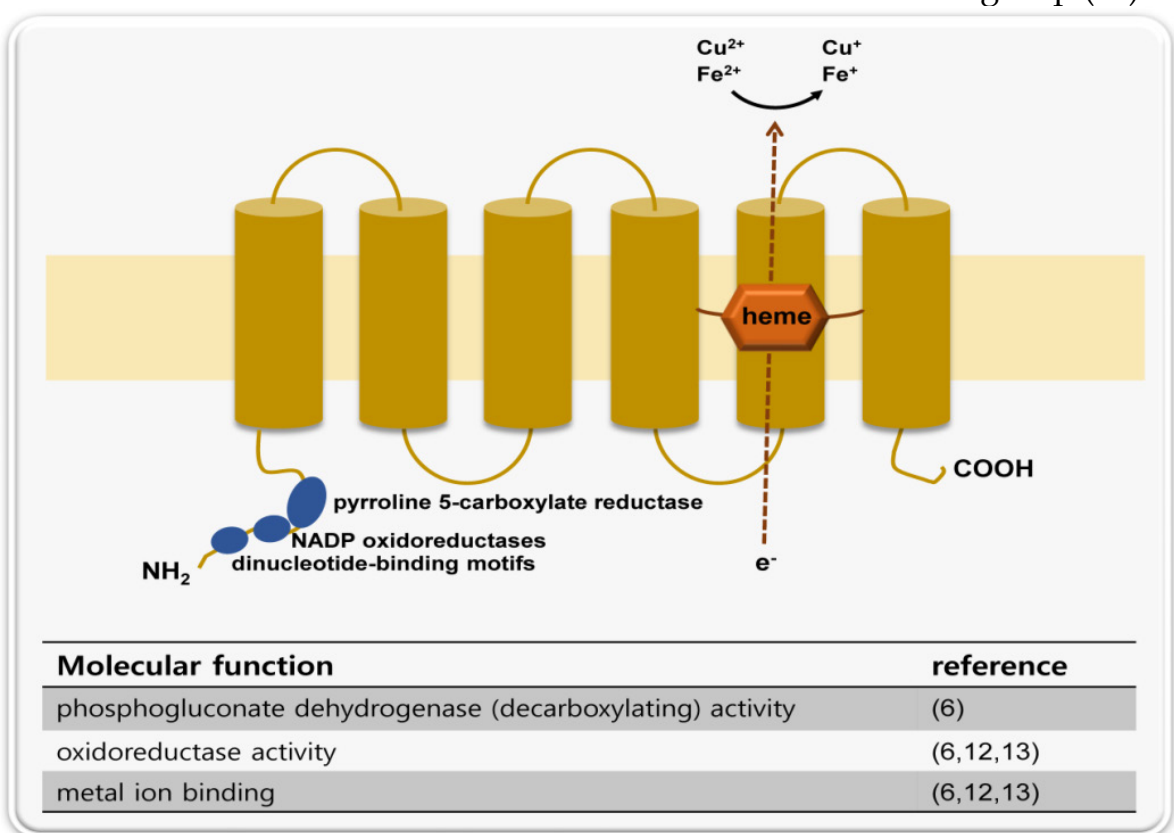

Figure I. A schematic representation of STAMP2 protein structure and molecular functions. STAMP2 is a six transmembrane protein. The $\mathrm{N}$-terminal domain contains three conserved motifs: a dinucleotide-binding motif, an oxidoreductase motif and a pyrroline 5-carboxylate reductase-like motif. In accordance with these structural features, STAMP2 has three molecular functions: metal ion binding, oxidoreductase activity and phosphogluconate dehydrogenase activity. 
The STAMP2 $\mathrm{Km}$ value is stable, even in the presence of various mutations. It is possible that the three dimensional structure, and not a single conserved residue, is important for the protein's activity. This is consistent with the Epidemiological Study of Insulin Resistance Syndrome (DESIR) results for STAMP2, which showed that 9 single nucleotide polymorphisms (SNPs) had little, if any, significant correlation with metabolic syndrome (14).

\section{STAMP2 localization}

Quantitative time-lapse and immunofluorescence confocal microscopy using STAMP2 labeled with an $\mathrm{N}$-terminal green fluorescent protein (GFP) tag showed that STAMP2 localizes predominantly to the plasma membrane. Notably, STAMP2 was also detected in cytoplasmic vesicles associated with the Golgi apparatus. Therefore, STAMP2 appears to localize to the Golgi complex, the trans-Golgi network, and the plasma membrane (3).

Another group assessed STAMP2 localization by transiently transfecting COS-1 cells with pEGFP-C1-STAMP2. Their results showed that GFP-STAMP2 overlap significantly with beta-COP (coat protein marker), giantin (mid-Golgi marker), and TGN-46 (TGN marker), three well-characterized Golgi markers.

Time-lapse confocal microscopy shows rapid trafficking of GFP-STAMP2 in the cytosol in vesiculotubular structures (VTS). Some of the VTSs move in curvilinear paths, and others move in straight paths (6). GFP-STAMP2 also co-localizes significantly with EEA1, an early endosome-associated protein (15). STAMP2 exhibits a fairly stable $\mathrm{K} m$ value under acidic conditions, which suggests that the protein could be active in intracellular organelles (13). Taken together, these data suggest that STAMP2 is associated with the secretory pathway, early endosomes, and the endocytic pathway. While its exact role in molecular trafficking is unknown, STAMP2 may reach the endoplasmic reticulum in an unfolded state and then acquire its active conformation. Alternatively, STAMP2 may have a significant role in trafficking (6).

Stamp2 co-localizes with caveolin-1 in 3T3-L1 adipocytes. Association with caveolin-1, and possibly with the caveolar signaling complex, may explain some of the TNF-a-mediated effects of STAMP2 (16).

\section{STAMP2, Inflammation and Metabolism}

STAMP2 expression increased dramatically in dose-dependent manner when differentiating adipocytes were exposed to TNF-a (3). In addition,
STAMP2 mRNA expression was stimulated by interleukin (IL)-6 in a dose-dependent and time-dependent fashion (17).

Wellen et al. identified STAMP2 through transcriptional profiling while searching for proteins that counter-regulate inflammatory signals in adipose tissue. They showed that Stamp2 expression in 3T3-L1 adipocytes was induced not only by TNF-a but also by nutritional signals such as high serum and fatty acids. Similar results were shown in in vivo models: Stamp2 expression was elevated in fed mice compared to fasting mice. This response was lost in ob/ob mice, as well as in mice rendered obese through a high-fat diet.

Loss-of-function experiments provided further insight. Loss of STAMP2 function both in vitro and in vivo resulted in elevated inflammatory markers, diminished insulin sensitivity, and dysfunctional glucose uptake. Stamp2 knockout mice also exhibited an increased number of macrophages (18). Based on these observations, many studies suggest that STAMP2 integrates inflammatory and nutritional signals with metabolism (Figure 2) $(19,20)$. STAMP2 may counter-regulate insulin resistance by regulating macrophage polarization in visceral adipose tissue (VAT) and BAT (21).

STAMP2 expression is high in human adipose tissue as well. Adipokines such as TNF- $\alpha$, IL-6, and leptin regulate STAMP2 expression.

However, some points remain unclear. For example, unlike TIARP, STAMP2 does not appear to influence human adipocyte differentiation (4). More importantly, there are conflicting data regarding STAMP2 expression in subcutaneous and visceral adipose tissues $(7,10,22)$. More research is needed to resolve these discrepancies.

The anti-inflammatory activity of STAMP2 has been shown in other tissues as well, most notably the liver. The transcription factor STAT3 is well known for negatively regulating hepatic gluconeogenic gene expression, thus playing a protective role in hepatic insulin signaling (23). Hepatic STAMP2 appears to be a bona fide STAT3 target (24). This suggests that STAMP2 exerts a protective role in maintaining hepatic insulin signaling in the presence of inflammation and obesity signals. STAMP2 was also found to control macrophage inflammation by controlling NADPH homeostasis (25).

The importance of STAMP2 in inflammation and metabolism appears to be conserved, although the regulatory mechanism differs by species and tissue. Future research should address these differences. 


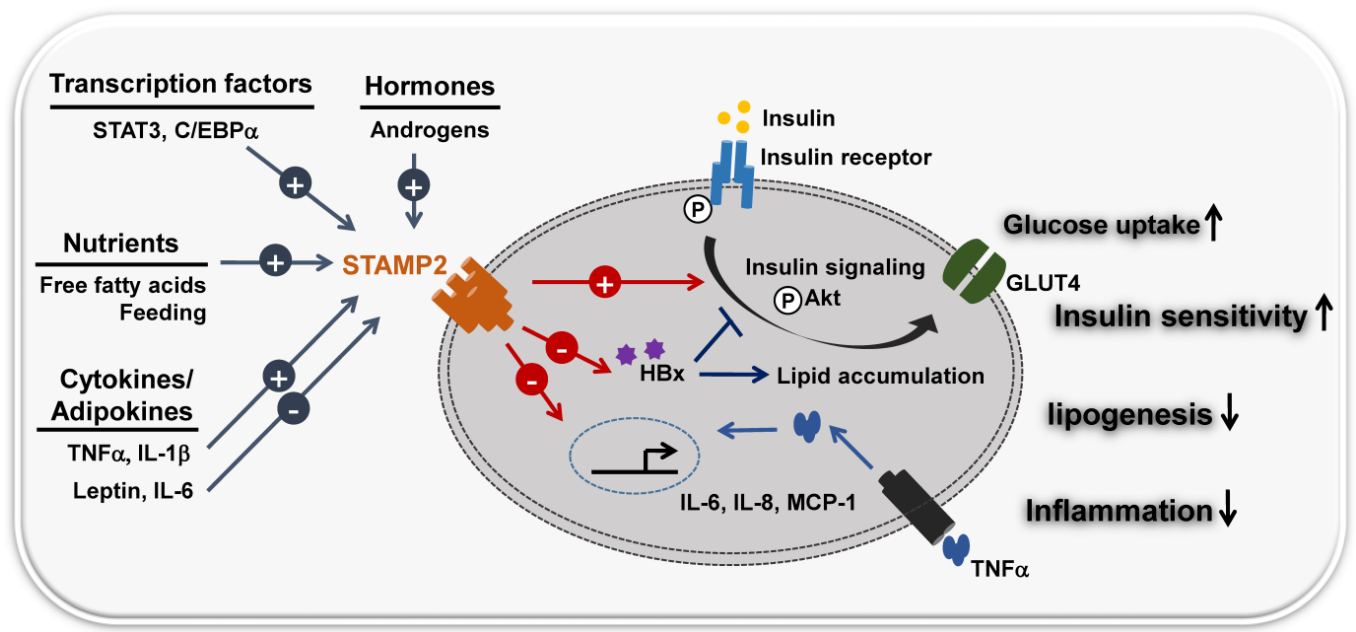

Figure 2. STAMP2 links inflammation and metabolism. STAMP2 integrates nutrient, hormone and inflammatory signals with metabolic pathways. FFA/feeding stimuli and inflammatory signals (cytokines)/adipokines (TNF- $\alpha$, IL-6, IL-8, leptin) modulate the expression of the transmembrane protein STAMP2. IL-6 and leptin (repressors) and TNF- $\alpha$ and IL-I $\beta$ (inducers) regulate STAMP2 metabolic activity, similar to the insulin signaling pathway. STAMP2 up-regulates insulin sensitivity by increasing tyrosine phosphorylation of both the insulin receptor (IR) and Akt kinase, translocation of the glucose transporter GLUT4 to the plasma membrane, and glucose uptake. Hepatitis B virus $X$ protein $(\mathrm{HBx})$ causes metabolic disorders including insulin resistance and abnormal lipid accumulation in the liver. STAMP2 antagonizes HBx-mediated hepatocyte dysfunction by decreasing HBx protein stability.

\section{STAMP2 and prostate cancer}

Ironically, it is still unknown how STAMP2 is associated with prostate cancer. There is no evidence of STAMP2 induction by TNF- $a$ in human prostate cancer cells nor do androgens stimulate Stamp2 in cultured murine adipocytes (26).

Several studies have shed light on this association of STAMP2 with prostate cancer. STAMP2 overexpression in the PC-3 cell line resulted in an increase in cell number and size. STAMP2 overexpression in COS7 and DU145 cells increased cell growth (6). This is consistent with the fact that STEAP mRNA in general seems to be overexpressed in solid tumors but undetectable in in non-malignant tumors (27).

STAMP2 is tightly regulated by androgens in the AR-positive prostate cancer cell line LNCaP, but not in androgen receptor-negative cell lines such as PC3, Du145, CAHPV10, PZ-HPV7, and YPEN-1 $(6,26,28)$. This differential expression pattern may contribute to the malignancy of prostate cancer. CpG islands were detected next to the STAMP2 promoter region in LNCaP and DU145 cells, but were only methylated in the DU145 cell line. STAMP2 expression was restored in DU145 cells when the methylation was reversed (29). Further research is needed to elucidate the biological significance of this epigenetic trait.

STAMP2 has opposite effects on proliferation in anchorage-dependent and anchorage-independent cell lines. One study indicated that this difference is due to differential phosphorylation of focal adhesion kinase (FAK). STAMP2 overexpression correlates with reduced FAK phosphorylation in anchorage-independent cells. Inadequate FAK phosphoryla- tion may then lead to reduced cell growth (29).

It is reasonable to expect that the growth-inducing and anti-apoptotic roles of STAMP2 are required for carcinogenesis (30). This would explain why STAMP2 is overexpressed in androgen receptor-positive prostate cancer. As the cancer progresses, it may gain additional mutations, which themselves are sufficient to produce malignancy, even without STAMP2. This multiple-hit model could explain why androgen receptor-negative cell lines do not express STAMP2. On the other hand, it is also possible that increased STAMP2 expression is due to various nutritional conditions caused by prostate cancer pathology. More research is required to fully understand this issue. Currently, the most fitting clinical application for STAMP2 is as a biomarker for prostate cancer malignancy $(26,28)$.

\section{STAMP2 and Mitochondria}

The inhibitory role of STAMP2 towards inflammation and metabolism has been widely investigated, but the underlying mechanism is not fully understood. Because the most prominent function of STAMP2 is its metalloreductase activity, it is likely that this activity is responsible for inhibiting inflammation and metabolic activity.

Metalloreductase is involved in numerous biological processes: (i) reducing metals for cellular uptake; (ii) modulating oxidative stress; and (iii) regulating co-enzyme (in this case, for NADPH) in metabolism regulation. Several studies suggest STAMP2 performs all of these functions $(25,31,32)$. Interestingly, many STAMP2 features are related to mitochondria. Mitochondria are the major consumer of 
cellular iron, as they utilize heme and iron-sulfur clusters. Iron is also an important element in the electron transport chain (33) (i.e., metal reduction). The electron transport chain forms reactive oxygen species (ROS) (i.e., modulating oxidative stress). NADPH is a major component of the electron transport chain (i.e., metabolism regulation). Moreover, the localization of STAMP2 in the ER, an organelle that engages in dynamic with mitochondria, may imply an intimate functional connection (34).

Although the mechanisms are not fully understood, many STAMP2-related pathologies are relevant to biological dysfunctions associated with mitochondria. Oxidative stress and inflammatory signals in mitochondria are thought to induce metabolic syndrome and insulin resistance $(35,36)$. Mitochondria are also thought to be a major prognosis factor for prostate cancer, as prognosis is related to PSA levels, androgen dependency, and growth in the bone marrow stromal environment (37-39). The hepatitis B virus $X$ protein $(\mathrm{HBx})$ is known to cause metabolic disorders and apoptosis by localizing to and targeting mitochondria (40-42). STAMP2 antagonizes HBx-mediated hepatocyte dysfunction. In addition, a physical interaction between HBx and STAMP2 has been confirmed by co-immunoprecipitation assays and fluorescence microscopy (43). Thus, we can cautiously presume that STAMP2 engages in cross-talk with the mitochondrial functional network.

STAMP2 may interact with mitochondria through several pathways, such as ROS level attenuation, signal transduction, and apoptosis. Zhou et al. demonstrated that STAMP2 plays a critical role in in vitro osteoclastogenesis by regulating cellular iron and ROS levels, thus affecting mitochondrial biogenesis (32). Treating mature adipocytes with a monoclonal antibody against STAMP2 resulted in attenuated phosphorylation of IRS-1, P13K, and Akt, which are known upstream components of mitochondrial signaling pathways (44). Additionally, treating cells with a monoclonal antibody against STAMP2 promotes the appearance of apoptotic cellular morphology and mitochondrial damage, possibly mediated by caspase-3 and caspase-8 (30). Future studies of the STAMP2-mitochondrial network will shed light on poorly-understood features of STAMP2 function.

\section{Concluding remarks}

STAMP2 is a novel protein whose function is not well understood. As discussed above, human STAMP2 mRNA expression is significantly up-regulated in prostate tumors compared to normal glands, and STAMP2 overexpression can induce cell proliferation. This suggests that STAMP2 could be used as a prostate cancer marker. The STAMP2 pro- tein has metalloreductase activity, which may be important for STAMP2 function. STAMP2 gene expression is also highly correlated with insulin resistance and inflammation, which could help support the hypothesis that inflammation in metabolic tissues contributes to the development of insulin resistance. Despite its importance, the molecular mechanisms of STAMP2 function are not well understood. The structural and functional features of STAMP2 strongly suggest that it plays a key role in mitochondrial biology. Further research is needed to understand the biological significance of STAMP2.

\section{Abbreviations}

STAMP2: six transmembrane protein of prostate 2; STEAP: six transmembrane epithelial antigen of the prostate; TIARP: tumor necrosis factor a-induced adipose-related protein; TNF- $\alpha$ : Tumor necrosis factor-alpha.

\section{Acknowledgements}

This work was supported by the National Research Foundation of Korea (NRF) grant funded by the Korea government (MSIP) (No. 2013 041811).

\section{Competing Interests}

The authors have declared that no competing interest exists.

\section{References}

1. Hubert RS, Vivanco I, Chen E, Rastegar S, Leong K, Mitchell SC, Madraswala R, Zhou Y, Kuo J, Raitano AB. STEAP: A prostate-specific cell-surface antigen highly expressed in human prostate tumors. Proceedings of the National Academy of Sciences. 1999;96(25):14523-8.

2. Korkmaz KS, Elbi C, Korkmaz CG, Loda M, Hager GL, Saatcioglu F. Molecular cloning and characterization of STAMP1, a highly prostate-specific six transmembrane protein that is overexpressed in prostate cancer. J Biol Chem. 2002;277(39):36689-96.

3. Moldes M, Lasnier F, Gauthereau X, Klein C, Pairault I, Fève B, Chambaut-Guérin A. Tumor necrosis factor-a-induced adipose-related protein (TIARP), a cell-surface protein that is highly induced by tumor necrosis factor- $\alpha$ and adipose conversion. J Biol Chem. 2001;276(36):33938-46.

4. Chen X, Zhu C, Ji C, Zhao Y, Zhang C, Chen F, Gao C, Zhu J, Qian L, Guo X. STEAP4, a gene associated with insulin sensitivity, is regulated by several adipokines in human adipocytes. Int J Mol Med. 2010;25(3):361-7.

5. Jemal A, Siegel R, Ward E, Hao Y, Xu J, Murray T, Thun MJ. Cancer statistics, 2008. CA: a cancer journal for clinicians. 2008;58(2):71-96.

6. Korkmaz CG, Korkmaz KS, Kurys P, Elbi C, Wang L, Klokk TI, Hammarstrom C, Troen G, Svindland A, Hager GL. Molecular cloning and characterization of STAMP2, an androgen-regulated six transmembrane protein that is overexpressed in prostate cancer. Oncogene. 2005;24(31):4934-45.

7. ZHANG C, Chi X, Wang B, Zhang M. Downregulation of STEAP4, a highly-expressed TNF- $\alpha$-inducible gene in adipose tissue, is associated with obesity in humans1. Acta Pharmacol Sin. 2008;29(5):587-92.

8. Inoue A, Matsumoto I, Tanaka Y, Iwanami K, Kanamori A, Ochiai N, Goto D, Ito S, Sumida T. Tumor necrosis factor alpha-induced adipose-related protein expression in experimental arthritis and in rheumatoid arthritis. Arthritis Res Ther. 2009;11(4):R118.

9. Sikkeland J, Saatcioglu F. Differential expression and function of stamp family proteins in adipocyte differentiation. PloS one. 2013;8(7):e68249.

10. Arner P, Stenson BM, Dungner E, Näslund E, Hoffstedt J, Ryden M, Dahlman I. Expression of six transmembrane protein of prostate 2 in human adipose tissue associates with adiposity and insulin resistance. Journal of Clinical Endocrinology \& Metabolism. 2008;93(6):2249-54.

11. Ohgami RS, Campagna DR, Greer EL, Antiochos B, McDonald A, Chen J, Sharp JJ, Fujiwara Y, Barker JE, Fleming MD. Identification of a ferrireductase required for efficient transferrin-dependent iron uptake in erythroid cells. Nat Genet. 2005;37(11):1264-9. 
12. Ohgami RS, Campagna DR, McDonald A, Fleming MD. The steap proteins are metalloreductases. Blood. 2006;108(4):1388-94.

13. Gauss GH, Kleven MD, Sendamarai AK, Fleming MD, Lawrence CM. The crystal structure of six transmembrane antigen of the prostate 4 (Steap4), a ferri/cuprireductase, suggests a novel inter-domain flavin binding site. J Biol Chem. 2013.

14. Miot A, Maimaitiming S, Emery N, Bellili N, Roussel R, Tichet J, Velho G, Balkau B, Marre M, Fumeron F. Genetic variability at the six transmembrane protein of prostate 2 locus and the metabolic syndrome: The data from an epidemiological study on the insulin resistance syndrome (DESIR) study. Journal of Clinical Endocrinology \& Metabolism. 2010;95(6):2942-7.

15. Stenmark H, Aasland R, Toh B, D'Arrigo A. Endosomal localization of the autoantigen EEA1 is mediated by a zinc-binding FYVE finger. J Biol Chem. 1996;271(39):24048-54.

16. Chambaut-Guérin A, Pairault J. Tumour necrosis factor a-induced adipose-related protein (TIARP): Co-localization with caveolin-1. Biology of the Cell. 2005;97(5):339-47.

17. Fasshauer M, Kralisch S, Klier M, Lossner U, Bluher M, Chambaut-Guérin A, Klein J, Paschke R. Interleukin-6 is a positive regulator of tumor necrosis factor a-induced adipose-related protein in 3T3-L1 adipocytes. FEBS Lett. 2004:560(1):153-7.

18. Wellen KE, Fucho R, Gregor MF, Furuhashi M, Morgan C, Lindstad T, Vaillancourt E, Gorgun CZ, Saatcioglu F, Hotamisligil GS. Coordinated regulation of nutrient and inflammatory responses by STAMP2 is essential for metabolic homeostasis. Cell. 2007;129(3):537-48.

19. Waki H, Tontonoz P. STAMPing out inflammation. Cell. 2007;129(3):451-2.

20. Abedini A, Shoelson SE. Inflammation and obesity: STAMPing out insulin resistance? Immunol Cell Biol. 2007;85(6):399-400.

21. Han L, Tang M, Ti Y, Wang Z, Wang J, Ding W, Wang H, Zhang Y, Zhang W, Zhong M. Overexpressing STAMP2 improves insulin resistance in diabetic ApoE-/-/LDLR-/- mice via macrophage polarization shift in adipose tissues. PloS one. 2013;8(11):e78903.

22. Moreno-Navarrete JM, Ortega F, Serrano M, Pérez-Pérez R, Sabater M, Ricart W, Tinahones F, Peral B, Fernández-Real JM. Decreased STAMP2 expression in association with visceral adipose tissue dysfunction. Journal of Clinical Endocrinology \& Metabolism. 2011;96(11):E1816-25.

23. Inoue $\mathrm{H}$, Ogawa $W$, Ozaki M, Haga S, Matsumoto M, Furukawa K, Hashimoto N, Kido Y, Mori T, Sakaue H. Role of STAT-3 in regulation of hepatic gluconeogenic genes and carbohydrate metabolism in vivo. Nat Med. 2004;10(2):168-74.

24. Ramadoss $\mathrm{P}$, Chiappini F, Bilban M, Hollenberg AN. Regulation of hepatic six transmembrane epithelial antigen of prostate 4 (STEAP4) expression by STAT3 and CCAAT/enhancer-binding protein a. J Biol Chem. 2010;285(22):16453-66.

25. ten Freyhaus H, Calay ES, Yalcin A, Vallerie SN, Yang L, Calay ZZ, Saatcioglu F, Hotamisligil GS. Stamp2 controls macrophage inflammation through nicotinamide adenine dinucleotide phosphate homeostasis and protects against atherosclerosis. Cell Metabolism. 2012;16(1):81-9.

26. Lindstad T, Jin Y, Wang L, Qu S, Saatcioglu F. STAMPs at the crossroads of cancer and nutrition. Nutr Cancer. 2010;62(7):891-5.

27. Valenti M, Dalle Carbonare L, Donatelli L, Bertoldo F, Giovanazzi B, Caliari F, Lo Cascio V. STEAP mRNA detection in serum of patients with solid tumours. Cancer Lett. 2009;273(1):122-6.

28. Gomes IM, Maia CJ, Santos CR. STEAP proteins: From structure to applications in cancer therapy. Molecular Cancer Research. 2012;10(5):573-87.

29. Tamura T, Chiba J. STEAP4 regulates focal adhesion kinase activation and CPG motifs within STEAP4 promoter region are frequently methylated in DU145, human androgen-independent prostate cancer cells. Int J Mol Med. 2009;24(5):599-604.

30. Qin D, Kou C, Ni Y, Zhang C, Zhu J, Zhu C, Wang Y, Zhu G, Shi C, Ji C. Monoclonal antibody to the six-transmembrane epithelial antigen of prostate 4 promotes apoptosis and inhibits proliferation and glucose uptake in human adipocytes. Int J Mol Med. 2010;26(6):803.

31. Wellen KE, Fucho R, Gregor MF, Furuhashi M, Morgan C, Lindstad T, Vaillancourt E, Gorgun CZ, Saatcioglu F, Hotamisligil GS. Coordinated regulation of nutrient and inflammatory responses by STAMP2 is essential for metabolic homeostasis. Cell. 2007;129(3):537-48.

32. Zhou J, Ye S, Fujiwara T, Manolagas SC, Zhao H. Steap4 plays a critical role in osteoclastogenesis in vitro by regulating cellular iron/reactive oxygen species (ROS) levels and cAMP response element-binding protein (CREB) activation. J Biol Chem. 2013 Oct 18;288(42):30064-74.

33. Richardson DR, Lane DJ, Becker EM, Huang ML, Whitnall M, Rahmanto YS, Sheftel AD, Ponka P. Mitochondrial iron trafficking and the integration of iron metabolism between the mitochondrion and cytosol. Proceedings of the National Academy of Sciences. 2010;107(24):10775-82.

34. Giorgi C, De Stefani D, Bononi A, Rizzuto R, Pinton P. Structural and functional link between the mitochondrial network and the endoplasmic reticulum. Int J Biochem Cell Biol. 2009;41(10):1817-27.

35. Kim J, Wei Y, Sowers JR. Role of mitochondrial dysfunction in insulin resistance. Circ Res. 2008;102(4):401-14.

36. Martínez JA. Mitochondrial oxidative stress and inflammation: An slalom to obesity and insulin resistance. J Physiol Biochem. 2006;62(4):303-6.

37. Higuchi M, Kudo T, Suzuki S, Evans T, Sasaki R, Wada $Y$, Shirakawa T, Sawyer J, Gotoh A. Mitochondrial DNA determines androgen dependence in prostate cancer cell lines. Oncogene. 2005;25(10):1437-45.
38. Arnold RS, Sun CQ, Richards JC, Grigoriev G, Coleman IM, Nelson PS, Hsieh C, Lee JK, Xu Z, Rogatko A. Mitochondrial DNA mutation stimulates prostate cancer growth in bone stromal environment. Prostate. 2009;69(1):1-11.

39. Kloss-Brandstätter A, Schäfer G, Erhart G, Hüttenhofer A, Coassin S, Seifarth C, Summerer M, Bektic J, Klocker H, Kronenberg F. Somatic mutations throughout the entire mitochondrial genome are associated with elevated PSA levels in prostate cancer patients. The American Journal of Human Genetics. 2010;87(6):802-12.

40. Clippinger AJ, Bouchard MJ. Hepatitis B virus HBx protein localizes to mitochondria in primary rat hepatocytes and modulates mitochondrial membrane potential. J Virol. 2008;82(14):6798-811.

41. Lim W, Kwon S, Cho H, Kim S, Lee S, Ryu W, Cho H. HBx targeting to mitochondria and ROS generation are necessary but insufficient for HBV-induced cyclooxygenase- 2 expression. Journal of molecular medicine. 2010;88(4):359-69.

42. Henkler F, Hoare J, Waseem N, Goldin RD, McGarvey MJ, Koshy R, King IA. Intracellular localization of the hepatitis B virus HBx protein. J Gen Virol. 2001;82(4):871-82.

43. Kim HY, Cho HK, Yoo SK, Cheong J. Hepatic STAMP2 decreases hepatitis B virus $X$ protein-associated metabolic deregulation. Exp Mol Med. 2012;44(10):622-32.

44. Qin D, Zhu J, Ji C, Kou C, Zhu G, Zhang C, Wang Y, Ni Y, Guo X. Monoclonal antibody to six transmembrane epithelial antigen of prostate-4 influences insulin sensitivity by attenuating phosphorylation of P13K (P85) and akt: Possible mitochondrial mechanism. J Bioenerg Biomembr. 2011;43(3):247-55. 